\title{
Fatores de risco associados à prática de atividade física de universitários no início e final da graduação
}

http://dx.doi.org/10.11606/1807-5509201800040547

\author{
Guilherme da Silva GASPAROTTO* \\ Wagner de CAMPOS**
}

*Instituto Federal de

Educação, Ciência e

Tecnologia do Paraná,

Curitiba, PR, Brasil.

**Departamento de

Educação Física,

Universidade Federal

do Paraná, Curitiba

PR, Brasil.

\section{Resumo}

Este estudo de delineamento descritivo, longitudinal, com 1.197 universitários se propôs a verificar: a) possibilidade de alteração na prática de atividade física de estudantes universitários após quatro anos de curso; b) associação entre a prática de atividade física moderada a vigorosa (AFMV) e atividade física vigorosa (AFV) com fatores de risco cardiovascular (FRC) avaliados no início e fim da graduação; c) fração atribuída à prática de AFMV e AFV na associação com FRC. Por meio dos questionários International Physical Activity Questionnaire e Youth Risk Behavior Survey, foram respectivamente obtidas medidas da prática de atividade física e dos seguintes FRC: consumo de bebidas alcoólicas, tabagismo e hábitos alimentares. Além desses fatores, foram verificados: pressão arterial, IMC e circunferência da cintura (CC). Pela Razão de Prevalência identificou-se associação da AFMV e AFV com os FRC. Calculou-se também a fração atribuída populacional (FAP) da atividade física sobre os FRC. Foi verificada manutenção da prática de AFMV, 46,4\% em 2011 e 50,3\% em 2014, bem como de AFV, 30,7\% em 2011 e 35,2\% em 2014. Além disso, observou-se relação positiva da prática de atividade física com consumo de bebidas alcoólicas e de frutas, saladas e/ou vegetais, além de relação protetiva com a CC elevada, em 2011 e 2014 ( $p<0,05)$. A prática de atividade física apresentou associação com alguns FRC nos dois periodos avaliados. Entre os mais associados estiveram o consumo recomendado de frutas, saladas e/ou vegetais e a circunferência de cintura elevada. Outros fatores de risco podem estar associados com diferentes variáveis socioambientais.

Palavras-Chave: Estudantes; Exercício; Fatores de Risco; Estilo de Vida.

\section{Introdução}

É sabido que grande parcela das mortes no mundo é causada por doenças cardiovasculares (DCVs). Segundo a Organização Mundial da Saúde (OMS) ${ }^{1}$, doenças como Infarto Agudo do Miocárdio (IAM), Insuficiência Cardíaca (IC), Acidente Vascular Encefálico (AVE), entre outras somam 30\% da mortalidade mundial.

Entre os diversos fatores de risco, podem-se observar os de ordem comportamental (utilizaçáo de substâncias nocivas, como álcool e tabaco, hábitos alimentares inadequados, prática insuficiente de atividades físicas) e metabólica, associadas ou não à predisposição genética (obesidade, hiperglicemia, hipertrigliceridemia, hipertensão arterial $)^{2-5}$.
Apesar das DCVs, especificamente, não serem comumente observadas entre populações jovens, muitos fatores de risco cardiovasculares (FRC) podem ser desenvolvidos ou adquiridos nas primeiras décadas de vida ${ }^{6}$. Nesta perspectiva, estudantes universitários correspondem a uma relevante população de estudo, visto que, neste momento, o indivíduo passa por importantes modificaçóes no estilo de vida. Estas mudanças podem favorecer o desenvolvimento de DCVs em curto ou longo prazo ${ }^{7,8}$.

Entre os FRC relacionados ao comportamento, os mais comumente observados na população de estudantes são a utilização de tabaco, o consumo de álcool, os maus hábitos alimentares e a prática 
insuficiente de atividades físicas. Outros fatores são os indicadores de obesidade e pressão arterial elevada ${ }^{2,3,4,9}$.

Visto que a associação protetiva entre a prática de atividades físicas e FRC mostra-se bem embasada na literatura, supóe-se que prática insuficiente pode responder parcialmente por modificaçóes em FRC durante o período universitário ${ }^{10}$. Nesta perspectiva, observar o estilo de vida do estudante no início do curso e sua evolução após os anos da graduação pode auxiliar na identificação de grupos vulneráveis. Esse processo pode contribuir para o adequado direcionamento de açóes preventivas.

Apesar de se observar estudos envolvendo FRC em amostras de estudantes universitários, no Brasil, não é grande o número de trabalhos com amostras representativas ${ }^{11-13}$. Este viés diminui a força das análises e consequentemente impede que conclusóes concretas sejam estabelecidas diante dos resultados apresentados.

$\mathrm{Na}$ América latina, não houve, nos últimos 10 anos, estudo que tenha acompanhado o nível de atividade física e fatores relacionados em amostra de alunos durante o período de graduação. Pesquisas com este delineamento permitem verificar a parcela do desfecho (FRC) atribuída a uma variável de exposição, neste caso a prática de atividade física.

Diante do exposto, o objetivo deste estudo foi verificar possível alteração na prática de atividade física de estudantes universitários brasileiros após quatro anos de curso, a associação entre a prática de atividade física moderada a vigorosa (AFMV) e atividade física vigorosa (AFV) com FRC avaliados no início e fim da graduação e a fração atribuída à prática de AFMV e AFV na sua associação com FRC.

\section{Materiais e Método}

\section{População e amostra}

Trata-se de um estudo com delineamento descritivo, longitudinal prospectivo, segundo Anstey e Hofer ${ }^{14}$, caracterizado pela observação de uma mesma amostra, pelo menos em dois pontos na linha do tempo. Neste caso, a primeira avaliação realizada em 2011 e a segunda em 2014.

A população em estudo consistiu em 5.059 estudantes ingressantes na Universidade Federal do Paraná, Brasil, no ano de 2011. O número amostral necessário foi obtido com utilização da calculadora do software EpiInfo ${ }^{\circ}$, levando-se em consideração parâmetros de estudo prévio, que apresentou amostra e variáveis similares: nível de confiança de 95\%; poder amostral de $80 \%$; prevalência de formandos insuficientemente ativos de $60 \%$ e prevalência de ingressantes insuficientemente ativos de $50 \%{ }^{15}$.

$\mathrm{O}$ processo de seleção por conglomerados se deu em dois níveis, número de matriculados em diferentes áreas de conhecimento (Humanas, Exatas e Biológicas) e estudantes do diurno e noturno, a amostra final foi dividida proporcionalmente de acordo com esses critérios. Devido ao desenho do estudo foi adotada correção por efeito de delineamento (deff) de 1,5 mais 10\% para possíveis perdas de dados, como sugerido por MEDRONHO ${ }^{16} \mathrm{e}$ utilizado por outros estudos com amostragem por conglomerados, totalizando o valor necessário final de 907 alunos $^{17-19}$.

\section{Instrumentos e procedimentos}

Para acompanhamento do nível de atividade física foi utilizado o IPAQ (International Physical Activity Questionnaire) versão curta, validado internacionalmente para populaçáo jovem adulta e traduzido para a língua portuguesa ${ }^{20}$. Baseado no tempo em AFMV ou AFV adquirido com a resposta do instrumento, os indivíduos foram classificados como Suficientemente Ativos ou Insuficientemente Ativos, segundo o ponto de corte de 150 minutos ou mais de AFMV ou 75 minutos de AFV por semana, como recomenda a $\mathrm{OMS}^{10}$.

Com intençáo de diminuir o efeito da amplitude no tempo em AF relatada pelos indivíduos, além da utilização do ponto de corte sugerido, para determinação dos parâmetros de AF da amostra, essa variável foi dividida em quatro categorias de 100 minutos, em suas intensidades Leve, Vigorosa e Sedentária.

Para obtenção dos comportamentos de tabagismo, etilismo e hábitos alimentares, foram utilizadas questôes específicas desses comportamentos contidas no Youth Risk Behavior Surveillance System (YRBSS), desenvolvido pelo Center for Disease Control and Prevention (CDC), previamente validado para 
universitários brasileiros e utilizado em outros estudos com população similar ${ }^{21,22}$.

Para o tabagismo, os alunos foram questionados se houve utilizaçáo de cigarros em pelo menos um dia dos últimos trinta.

Sobre o consumo de álcool, foram questionados se, nos últimos trinta dias, foi ingerida pelo menos uma dose de bebida alcoólica, e, nesse período, se foram consumidas cinco ou mais doses numa mesma ocasião.

Quanto aos hábitos alimentares, os alunos foram questionados se, no dia anterior à pesquisa, houve o consumo de frutas, saladas verdes e/ou vegetais, salgadinhos (chips ou similares) e bolos, tortas ou doces em geral e a quantidade de porçóes consumidas. O consumo de frutas, saladas e/ou vegetais foi categorizado em três porçóes ou mais para cada tipo desses alimentos, de acordo com o sugerido pelo U.S. Department of Agriculture e U.S. Departmentof Health and Human Services ${ }^{23}$.

A classificação socioeconômica dos alunos foi realizada por meio da metodologia da ABEP, Associação Brasileira de Empresas de Pesquisa ${ }^{24}$. Segundo a metodologia, os indivíduos foram distribuídos em classes A, B, C, D e E.

As medidas antropométricas foram realizadas de acordo com a proposta de HeYward e STOLARCZYK ${ }^{25}$.

Estatura: Foi utilizada uma fita métrica metálica portátil com resolução de $0,1 \mathrm{~cm}$. Foi realizada medida entre os planos que tangenciam o vértex e a planta dos pés, com o avaliado em posição ortostática, descalço, com a menor quantidade de roupa possível.

Massa Corporal: para determinação da massa corporal, foi utilizada uma balança portátil com resoluçáo de $0,1 \mathrm{Kg}$, seguindo os critérios de posição da estatura.

$O$ índice de massa corporal (IMC) foi definido pela relaçáo equivalente da massa corporal pela estatura ao quadrado, IMC = massa corporal / estatura ${ }^{2}$. Os alunos foram classificados como: subpeso $\leq 18,5 \mathrm{~kg} / \mathrm{m}^{2}$, eutróficos IMC: $18,6-25 \mathrm{~kg} / \mathrm{m}^{2}$, sobrepeso IMC: $25-29,9 \mathrm{~kg} / \mathrm{m}^{2} \mathrm{e}$ obeso: IMC $\geq 30 \mathrm{~kg} / \mathrm{m}^{21}$.

Circunferência da cintura: foi obtida com uma fita antropométrica metálica, sendo medida no plano horizontal, no ponto médio entre o ponto íleo-cristal e a última costela flutuante. Os pontos de corte adotados para a circunferência da cintura (CC) segundo a OMS foram: $\geq 94 \mathrm{~cm}$ para homens $\mathrm{e} \geq 80 \mathrm{~cm}$ para mulheres ${ }^{1}$.
Pressão arterial elevada: a Pressão Arterial Sistólica (PAS) e a Diastólica (PAD) foram verificadas por meio do método auscultatório, no braço esquerdo de cada avaliado, mediante utilização de um estetoscópio e de um esfigmomanômetro analógico (marca BD) calibrado com uma semana de antecedência ao início da coleta dos dados, que teve duraçáo de cinco meses. Os estudantes ficaram, por pelo menos cinco minutos, em repouso, sentados com as pernas descruzadas, pés apoiados no chão e dorso recostado na cadeira antes da mediçáo. O braço foi posicionado na altura do coração com a palma da mão voltada para cima e o cotovelo ligeiramente fletido. $\mathrm{O}$ manguito do esfigmomanômetro foi posicionado cerca de 2 a 3 $\mathrm{cm}$ da fossa cubital, e a campânula do estetoscópio sobre a artéria braquial sem compressão excessiva.

A pressão arterial elevada seguiu os pontos de corte sugeridos pela Organização mundial da Saúde, maior ou igual a $140 / 90 \mathrm{mmHg}^{1}$.

A presente pesquisa foi aprovada pelo Comitê de Ética em Pesquisa com seres humanos do setor de Ciências da Saúde da Universidade Federal do Paraná, estando de acordo com a resoluçấo n. 466/2012 do Conselho Nacional de Saúde, sob o registro CEP/SD: 1043.168.10.11.

\section{Tratamento estatístico}

Os dados foram tabulados e analisados com utilização do Statistical Package for the Social Sciences (SPSS versão 18.0). A normalidade dos dados foi verificada por meio do teste de KolgomorovSmirnov. Para a análise de prevalência das variáveis foi realizada distribuição de frequência com Intervalo do Confiança de $95 \%$. A comparação entre prevalências da prática de atividade física inicial (2011) e do último período da graduação (2014) foi verificada por meio do teste de Qui-Quadrado (Macnemar) para tendência (Mantel-Haenszel). A associação entre a prática de atividade física (AFMV e AFV) e os fatores de risco cardiovascular foi testada nos dois tempos (2011 e 2014) por meio da Razão de Prevalência (RP), utilizando a Regressão de Poisson com variância robusta. Para os fatores que se apresentaram associados à prática de $\mathrm{AF}$ foi verificada a Fração Atribuída Populacional (FAP). Por meio da FAP foi possível estimar a fração atribuída à exposiçáo (AFMV e AFV) no resultado da associaçáo às variáveis desfechos (FRC). O nível de significância estipulado para as análises foi de $\mathrm{p}<0,05$. 


\section{Resultados}

Dentre os 5.059 indivíduos elegíveis para compor a amostra, eram necessários 907 e foram avaliados 1.221 alunos em 2011, distribuídos entre as três áreas de estudo (Ciências Humanas, Exatas e Biológicas). Após a retirada dos questionários respondidos incorretamente ou incompletos, totalizaram 1.197 alunos que mostraram média de idade de $18,3( \pm 2,8)$ anos.

$\mathrm{Na}$ segunda etapa de avaliação, em 2014, daqueles avaliados inicialmente, 455 foram reavaliados. A perda amostral, comumente apresentada neste tipo de delineamento de estudo, ocorreu por alguns fatores: a) foi impossível de avaliar 351 indivíduos (46\%), devido não retorno da coordenação do curso após três contatos via $e$-mail, telefone e uma visita pessoalmente; b) desistiram do curso 283 indivíduos (38\%); c) 108 indivíduos (16\%) se negaram a participar ou não estiveram presentes nos dias da coleta.
A descrição da prevalência de fatores socioambientais, no início (2011) e no final (2014) do estudo, está exposta na TABELA 1.

Dentre as categorias de tempo em minutos, relativas ao tipo de $\mathrm{AF}$ (leve, moderada, vigorosa ou sedentária), a comparação entre as medidas inicial (2011) e final (2014) apresentou diferenças somente na prática de AF moderada e sedentária no fim de semana. Foi possível identificar diminuição da proporçáo de indivíduos que realizaram trezentos minutos ou mais de AF moderada. Também se observou aumento da proporção daqueles que passaram até 299 minutos sentados em um dia do fim de semana. A comparação entre as medidas inicial e final, das categorias em tempo de prática dos tipos de $\mathrm{AF}$, encontra-se na TABELA 2.

TABELA 1 - Descrição socioambiental da amostra no início (2011) e final do estudo (2014)

\begin{tabular}{|c|c|c|c|c|c|}
\hline \multirow[t]{2}{*}{ Variável } & $\%(n)$ & IC 95\% & $\%(n)$ & IC 95\% & $\mathbf{P}$ \\
\hline & \multicolumn{2}{|c|}{2011} & \multicolumn{2}{|c|}{2014} & \\
\hline Idade (anos) & & & & & $0,01^{*}$ \\
\hline$<18$ & $18,5 \%(222)$ & $16,4-20,7$ & - & & \\
\hline$\geq 18$ & $81,5 \%(975)$ & $79,3-83,6$ & $100 \%(455)$ & - & \\
\hline \multicolumn{6}{|l|}{ Sexo } \\
\hline Feminino & $50 \%(599)$ & $47,3-53$ & $56 \%(255)$ & $52,2-59-4$ & $0,03^{*}$ \\
\hline Masculino & $50 \%(598)$ & $47-52,7$ & $44 \%(200)$ & $40,8-48,3$ & \\
\hline \multicolumn{6}{|l|}{ Estado civil } \\
\hline Solteiro & $92,9 \%(1112)$ & $91,4-94,3$ & $89,8(406)$ & $83-94,1$ & 0,38 \\
\hline Casado & $7,1 \%(85)$ & $5,7-8,6$ & $10,2(49)$ & $7,2-14,6$ & \\
\hline \multicolumn{6}{|l|}{ Área de estudo } \\
\hline Humanas & $38 \%(455)$ & $35,3-40,9$ & $30,3 \%(138)$ & $26,1-34,8$ & $0,04^{*}$ \\
\hline Exatas & $37,3 \%(446)$ & $34,6-40,1$ & $44,1 \%(187)$ & $41,3-49,9$ & \\
\hline Biológicas & $24,7 \%(296)$ & $22,1-27,2$ & $25,6 \%(130)$ & $20,2-28,8$ & \\
\hline NSE & & & & & 0,69 \\
\hline$A$ e B & $75,1 \%(899)$ & $72,7-77,5$ & $69,1 \%(314)$ & $66,4-72$ & \\
\hline $\mathrm{C}$ & $22,7 \%(272)$ & $20,4-25,1$ & $27,7 \%(126)$ & $22,4-31,8$ & \\
\hline D e E & $2,2 \%(26)$ & $1,3-3,1$ & $3,2 \%(05)$ & $0,3-6,9$ & \\
\hline Residência & & & & & 0,41 \\
\hline Com pais e irmãos & $70,7 \%(846)$ & $68-73,2$ & $68 \%(309)$ & $61,9-75,5$ & \\
\hline Outros parentes & $6,6 \%(79)$ & $5,3-8,0$ & $4,2 \%(19)$ & $1,1-9,2$ & \\
\hline Cônjuge e filhos & $7,0 \%(84)$ & $5,6-8,5$ & $5,3 \%(25)$ & $1,2-11,1$ & \\
\hline Casa de estudantes & $8,6 \%(103)$ & $7,0-10,2$ & $14,2 \%(64)$ & $8,4-19,8$ & \\
\hline Casa de família (não parentes) & $2,2 \%(26)$ & $1,3-3,0$ & $2 \%(9)$ & $0,1-5,2$ & \\
\hline Sozinho & $4,9 \%(59)$ & $3,8-6,3$ & $6,3 \%(29)$ & $1,9-11,3$ & \\
\hline Período de estudo & & & & & 0,05 \\
\hline Manhã & $28,5 \%(341)$ & $25,8-31,1$ & $35,6 \%(162)$ & $28,4-40$ & \\
\hline Tarde & $7,6 \%(91)$ & $6,1-9,1$ & $3,3 \%(15)$ & $0,3-7,1$ & \\
\hline Noite & $25,3 \%(303)$ & $23,1-27,8$ & $20,5 \%(93)$ & $14,9-27,2$ & \\
\hline Integral & $38,6 \%(462)$ & $35,9-41,3$ & $40,6 \%(185)$ & $35,3-46,1$ & \\
\hline
\end{tabular}


TABELA 2 - Comparação das variáveis relacionadas à atividade física e sedentária

\begin{tabular}{|c|c|c|c|}
\hline \multirow{3}{*}{$\begin{array}{l}\text { Variáveis } \\
\text { Pratica de atividade física (minutos) }\end{array}$} & \multicolumn{2}{|c|}{ Ano } & \multirow{3}{*}{$\mathbf{p}$} \\
\hline & 2011 & 2014 & \\
\hline & $\%($ IC 95\%) & $\%($ IC 95\%) & \\
\hline Prática de AF leve caminhada & & & 0,224 \\
\hline $0-99$ & $37,6(34,9-40,3)$ & $43,4(37,9-48,6)$ & \\
\hline 100-199 & $20,6(18,3-22,8)$ & $18,7(14,7-23,0)$ & \\
\hline 200-299 & $15,9(13,9-18,0)$ & $15,8(12,1-19,8)$ & \\
\hline$\geq 300$ & $25,9(23,5-28,2)$ & $22,1(18,1-26,4)$ & \\
\hline Prática de AF moderada & & & $0,010^{*}$ \\
\hline $0-99$ & $56,6(53,6-59,6)$ & $60,6(55,5-65,2)$ & \\
\hline 100-199 & $17,2(15,0-19,4)$ & $21,3(17,0-25,9)$ & \\
\hline $200-299$ & $8,0(6,5-9,5)$ & $6,9(4,3-9,8)$ & \\
\hline$\geq 300$ & $18,1(16,0-20.5)$ & $11,2(8,0-14,7)^{*}$ & \\
\hline Prática de AF vigorosa & & & 0,278 \\
\hline $0-99$ & $71,4(68,9-73,9)$ & $67,5(62,6-72,1)$ & \\
\hline $100-199$ & $12,0(10,3-14,0)$ & $11,8(8,9-15,5)$ & \\
\hline $200-299$ & $4,1(2,9-5,3)$ & $6,0(3,7-8,6)$ & \\
\hline$\geq 300$ & $12,4(10,5-14,4)$ & $14,7(10,9-18,7)$ & \\
\hline Tempo sentado em um dia de semana & & & 0,750 \\
\hline $0-299$ & $10,5(8,8-12,3)$ & $11,5(8,1-14,9)$ & \\
\hline $300-599$ & $58,6(55,9-61,2)$ & $55,5(50,3-60,9)$ & \\
\hline $600-899$ & $28,2(25,5-30,7)$ & $30,5(25,6-35,1)$ & \\
\hline$\geq 900$ & $2,8(1,8-3,8)$ & $2,6(1,1-4,3)$ & \\
\hline Tempo sentado em um dia de fim de semana & & & $0,045^{*}$ \\
\hline $0-299$ & $30,0(27,3-32,8)$ & $36,8(31,9-42,0)^{*}$ & \\
\hline $300-599$ & $46,5(43,4-49,3)$ & $41,4(36,5-46,6)$ & \\
\hline $600-899$ & $18,6(16,4-20,7)$ & $18,7(14,7-22,7)$ & \\
\hline$\geq 900$ & $4,8(3,6-6,1)$ & $3,2(1,4-5,2)$ & \\
\hline
\end{tabular}

A prevalência de indivíduos que cumpriram a recomendação de prática de $\mathrm{AFMV}$ ou $\mathrm{AFV}$, definidas pela prática de no mínimo 150 ou 75 minutos por semana respectivamente, não apresentou diferença entre as avaliações de 2011 e 2014 . As comparaçôes das prevalências de cumpridores de AFMV e AFV estão apresentadas na FIGURA 1.

Quando testada comparação da prevalência dos que cumpriram a recomendação de AFMV e AFV entre os períodos de avaliação, utilizando somente os 455 indivíduos que realizaram as duas medidas, também não se observou alteração do número dos que cumpriram a recomendação. Estas comparações estão expostas na FIGURA 2.

A Razão de Prevalência, ajustada por sexo, idade, turno de estudo, NSE, tipo de residência e área de conhecimento, identificou associação entre a prática de AFMV: a) no primeiro momento do estudo, associação positiva com consumo excessivo de bebidas alcoólicas, associação positiva com o consumo de saladas e/ou vegetais; b) nos dois momentos do estudo houve associação positiva com o consumo de frutas, com o consumo recomendado de frutas, saladas e/ou vegetais concomitantemente, $\mathrm{e}$, como fator protetor para circunferência de cintura elevada.

Para essas variáveis que apresentaram associação, foi possível ainda, calcular a FAP, que mostrou a fração do resultado de desfecho atribuída à atividade física. Pelo resultado da FAP, percebeu-se contribuição importante da prática de atividade física, principalmente na medida realizada em 2014, na associação com o consumo recomendado de frutas, saladas e vegetais. Além de mostrar expressiva contribuição na associação protetiva da circunferência de cintura aumentada, em ambos os momentos avaliados. A análise ajustada de associação entre as categorias de tempo em prática de AFMV com os fatores de risco cardiovascular, bem como a FAP daqueles que apresentaram associação encontram-se na TABELA 3. 
Gasparotto GS, et al.

$\%$

PREVALÊNCIA DE AFMV E AFV

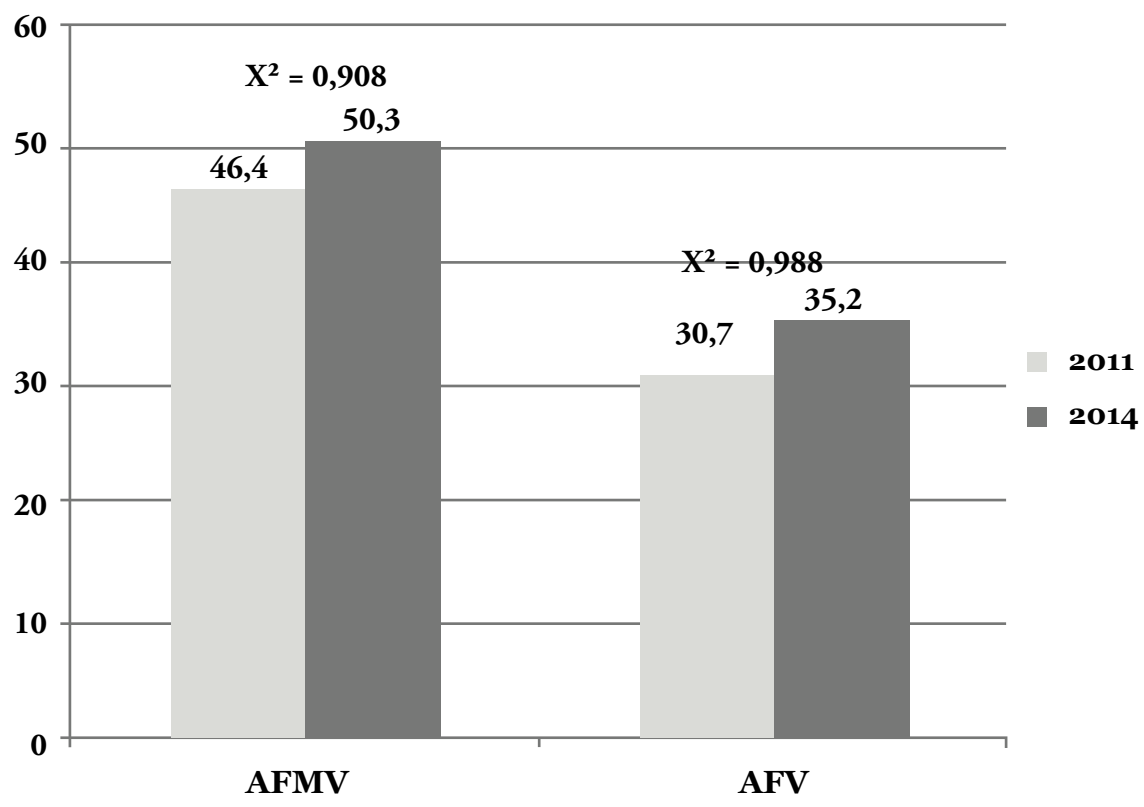

FIGURA 1 - Comparação da prevalência dos que cumpriram a recomendação de Atividade Física Moderado a Vigorosa e/ou Atividade Física Vigorosa (2011 - 2014).

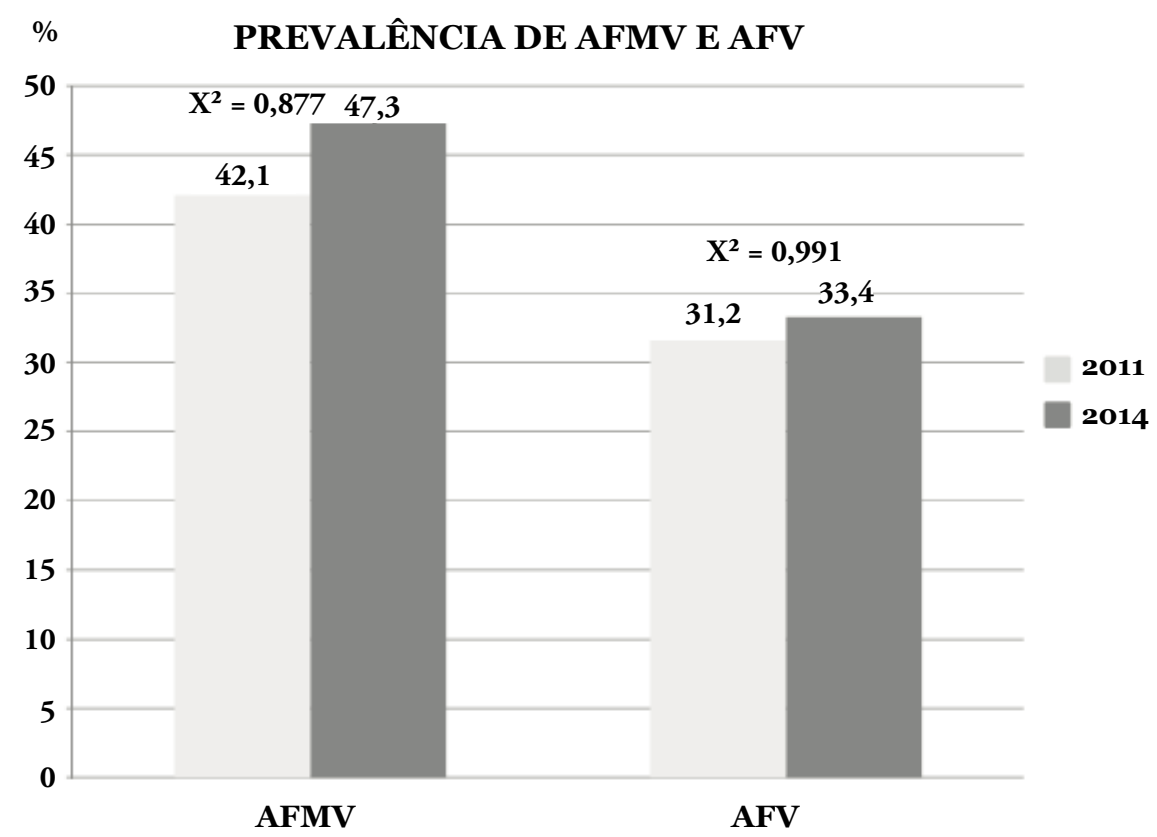

FIGURA 2 - Comparação da prevalência dos que cumpriram a recomendação de Atividade Física Moderado a Vigorosa e/ou Atividade Física Vigorosa (2011 - 2014), controlada pela participação nas duas medidas $(n=455)$. 
TABELA 3 - Razão de Prevalência ajustada da prática de AFMV e AFV (minutos semanais) para cada fator de risco e Fração atribuída Populacional de acordo com o ponto de corte recomendado

\begin{tabular}{lcccc}
\hline & \multicolumn{2}{c}{2011} & \multicolumn{2}{c}{2014} \\
\cline { 2 - 5 } Fator de risco & RP & FAP & RP & FAP \\
& $($ IC95\%) & $(\%)$ & $(I C 95 \%)$ & $(\%)$ \\
\hline
\end{tabular}

Fumou nos últimos 30 dias

AFMV $\geq 150$

$\mathrm{AFV} \geq 75$

Consumiu bebida alcoólica nos últimos 30 dias

AFMV $\geq 150$

$\mathrm{AFV} \geq 75$

Consumiu bebida alcoólica excessivamente nos últimos 30 dias
AFMV $\geq 150$
$1,20(1,02-1,46)^{*} \quad 6 \%$
$\mathrm{AFV} \geq 75$
$1,19(0,97-1,46)$
$1,19(0,85-1,67)$
$1,18(0,84-1,54)$

$1,19(0,82-1,75)$
$1,14(0,76-1,71)$

$0,51(0,27-1,01)$

$0,68(0,35-1,34)$
$1,13(0,98-1,31) \quad-$
$1,14(0,87-1,48)$
$1,11(0,85-1,47)$

Consumiu a recomendação de frutas no dia anterior
AFMV $\geq 150$
$1,69(1,12-2,57)^{*}$
18,7
$\mathrm{AFV} \geq 75$
$1,57(1,03-2,42)^{*}$
$14,2 \quad 3,64(1,84-7,21)^{*} \quad 43,8$

Consumiu a recomendação de saladas e/ou vegetais no dia anterior
AFMV $\geq 150$
$1,50(1,16-1,93)^{*}$
29,1
$1,32(0,87-2,03)^{*}$
$\mathrm{AFV} \geq 75$
$1,43(1,10-1,87)^{*}$
12,8
$1,92(1,26-2,94)^{*}$
29,0

Consumiu a recomendação de frutas, saladas e/ou vegetais no dia anterior

$$
\begin{aligned}
& \text { AFMV } \geq 150 \\
& \mathrm{AFV} \geq 75
\end{aligned}
$$

Consumiu salgados no dia anterior

AFMV $\geq 150$

$\mathrm{AFV} \geq 75$

Consumiu doces no dia anterior

AFMV $\geq 150$

$\mathrm{AFV} \geq 75$

Excesso de peso corporal

AFMV $\geq 150$

$\mathrm{AFV} \geq 75$

Circunferência de cintura aumentada

AFMV $\geq 150$

$\mathrm{AFV} \geq 75$

PAS Elevada

AFMV $\geq 150$

$\mathrm{AFV} \geq 75$

PAD Elevada

AFMV $\geq 150$

$\mathrm{AFV} \geq 75$

PA Elevada

AFMV $\geq 150$

$\mathrm{AFV} \geq 75$
$3,01(1,38-6,55)^{*} \quad 59,1 \quad 2,12(1,19-5,92)^{*} \quad 53,3$

$3,15(1,48-6,71)^{*} \quad 30,2 \quad 13,85(3,77-18,72)^{*} \quad 56,0$

$1,02(0,84-1,24) \quad-\quad 0,82(0,56-1,19)$

$1,04(0,83-1,29) \quad-\quad 0,76(0,51-1,41)$

$0,96(0,84-1,11) \quad-\quad 1,04(0,79-1,36)$

$0,96(0,83-1,12) \quad-\quad 0,92(0,68-1,23)$

$1,01(0,80-1,26) \quad-\quad 0,93(0,64-1,37)$

$0,94(0,73-1,21) \quad-\quad 0,88(0,57-1,29)$

$0,77(0,56-1,07)^{*} \quad 26,7 \quad 0,49(0,32-0,77)^{*} \quad 69,1$

$0,52(0,34-0,79)^{*} \quad 40,8 \quad 0,45(0,27-0,79)^{*} \quad 73,7$

$1,15(0,85-1,57) \quad-\quad 0,73(0,47-1,14)$

$1,02(0,73-1,42) \quad-\quad 0,67(0,42-1,08)$

$1,30(0,97-1,49) \quad-\quad 0,68(0,40-1,16)$

$0,82(0,61-1,09) \quad-\quad 0,75(0,42-1,31)$

$1,30(0,92-1,83) \quad-\quad 0,64(0,37-1,12)$

$0,94(0,56-1,24) \quad-\quad 0,59(0,32-1,08)$
RP: Razão de

Prevalência;

IC: Intervalo de

Confiança; FAP:

Fração Atribuída Populacional; PAS:

Pressão Arterial

Sistólica; PAD:

Pressão Arterial

Diastólica; PA:

Pressão Arterial;

${ }^{*} p<0,05$. 


\section{Discussão}

No presente estudo, a prevalência de indivíduos que cumpriram a recomendação mínima de AFMV e AFV não mostrou alteração ao fim do período de graduação. Entretanto, entre as categorias de AF moderada, foi identificada diferença nas prevalências de 2011 e 2014, principalmente em decorrência da diminuiçâoo de participantes de 300 ou mais minutos de atividade física moderada por semana. Segundo as recomendaçóes da OMS, atividades superiores a este ponto de corte (trezentos minutos) podem promover benefícios superiores na condição de saúde $e^{10}$.

A manutenção da quantidade de estudantes ativos surpreende, visto que, os estudos longitudinais que realizaram essa análise apontaram para diminuição da prática de atividade física após a entrada na universidade ${ }^{26,27}$. SMALL et al..$^{28}$ mostraram que, no início do curso, os estudantes completavam em média três dias e meio de no mínimo 30 minutos de AFMV. Já após sete semestres de curso, esses estudantes completaram no máximo dois dias com tais atividades. Os autores desses estudos citam que as principais justificativas apresentadas pelos estudantes são o acúmulo de tarefas com o passar do tempo na universidade e a falta de espaços públicos para prática de atividades dentro do campus e nas proximidades, fato relevante, já que os alunos passam boa parte do dia na universidade.

A OMS recomenda o mínimo de 150 minutos de AFMV ou 75 minutos de AFV semanais, com foco na promoção de saúde individual. Essas recomendaçóes são baseadas em diversos estudos que identificaram associação entre a prática desse tipo de atividade e fatores de risco à saúde ${ }^{10}$. Apesar da prevalência de indivíduos ativos, segundo as quantidades mínimas de AFMV e AFV sugeridas, manterem-se durante a graduação, a diminuiçáo de indivíduos que praticaram mais de 300 minutos de AF moderada semanalmente concorda com as referências supracitadas, do ponto de vista de diminuição na prática desse tipo de atividade.

No presente trabalho, entre as variáveis que não apresentaram relaçáo com a prática de AF esteve o consumo de cigarros. Outro estudo brasileiro, que se propôs a verificar a associação entre o nível de atividade física e tabagismo em universitários, também náo demonstrou tal relaçáa ${ }^{29}$. Esses autores sugerem que a baixa prevalência de consumo de tabaco dificulta a identificação da associação testada. Para tais pesquisadores, as incisivas açóes publicas promovidas para diminuição do tabagismo contribuíram efetivamente para esse fim nos últimos anos. Sabe-se que outros fatores se relacionam de forma mais intensa com este comportamento, como o baixo nível de escolaridade e o baixo nível socioeconômico, pouco prevalentes nesta população ${ }^{30}$.

Outra variável que não se relacionou com a prática de AF foi a PA. A prevalência deste fator de risco entre universitários também é baixa e pode influenciar no resultado da relação, como já identificado na literatura?

Neste estudo, a prática de AFMV acima de 150 minutos apresentou associação com o consumo de bebidas alcoólicas, somente no primeiro momento da pesquisa. Como observado, a prevalência de indivíduos que cumpriram a recomendação de prática de AFMV manteve-se durante o período, entretanto, o consumo de bebidas alcoólicas aumentou no mesmo período. Baseado na sensível associação observada pela FAP, pode-se sugerir que no segundo momento do estudo o elevado consumo de bebidas alcoólicas passou a estar mais associado a outros fatores ambientais ${ }^{30}$.

Apesar de a literatura indicar, em alguns casos, a existência de associação entre a intensidade das atividades físicas praticadas com o consumo de bebidas alcoólicas em populaçóes jovens, o mesmo não ocorreu nesta pesquisa ${ }^{5,31}$.

A prática de AFV não apresentou associação com o consumo de bebidas alcoólicas em suas categorias de tempo. Uma possível explicação para divergência entre os estudos, é que trabalhos internacionais, em que se identificou essa associação, foi utilizada prática esportiva para considerar AF vigorosa. Já o instrumento com o qual se mediu essa variável no presente estudo, o IPAQ, considera outros tipos de atividades diárias para somatória das AF vigorosas. A relação da prática esportiva com consumo de bebidas alcoólicas por jovens adultos é retratada com frequência na literatura ${ }^{32}$.

A relação entre a prática de atividade física, moderada ou vigorosa, com o excesso de peso corporal medido pelo IMC entre universitários não é clara. Diferentemente do que se espera, alguns estudos não apresentam tal associaçáa ${ }^{33,34}$. Neste trabalho, não foi observada associaçáo entre a prática de AFMV e AFV. Esse resultado está de acordo com outros estudos recentes, realizados com universitários brasileiros, que também não identificaram tal associação ${ }^{35,36}$. 
Apesar de não ter sido verificada relação entre a prática de $\mathrm{AF}$ e o excesso de peso corporal neste estudo, foi possível observar uma importante associação protetiva entre a prática de AFMV e AFV com a $\mathrm{CC}$ em ambos os momentos avaliados, em que as atividades físicas apresentam-se responsáveis por mais de $2 / 3$ do resultado da CC. Esse resultado apresentase relevante, pois alguns estudos demonstram que a centralizaçáo da gordura corporal pode predizer de forma efetiva doenças cardiovasculares ${ }^{37}$.

Entre universitários brasileiros, a medida de CC apresentou associaçóes mais importantes do que o IMC para glicemia elevada e HDL-c diminuído ${ }^{38}$. Assim, a prática de AF apresenta-se, de forma indireta, como relevante forma preventiva desses fatores de risco cardiovascular nesta população.

Este estudo apresentou algumas limitaçôes. A primeira diz respeito à perda de amostra na segunda onda de avaliação dos estudantes em 2014, em que participaram aproximadamente $40 \%$ dos universitários inicialmente avaliados e $60 \%$ do necessário, segundo cálculo amostral. Essa perda se deu principalmente pela falta de informaçóes dos egressos nas secretarias da universidade. Outra limitação, frequentemente discutida pela literatura, é a utilizaçáo de instrumento auto-relatado para coleta das informaçóes da variável independente do estudo, a prática de AF. Entretanto, deve-se ressaltar que, diante do tamanho, a avaliação de toda amostra de forma direta despenderia muito recurso financeiro e tempo para execução, o que inviabilizaria a pesquisa. Por fim, a primeira avaliação foi realizada durante todo o primeiro semestre de 2011 e a segunda durante todo o ano de 2014.
Logo, há a possibilidade de alguns alunos terem um intervalo de quase quatro anos entre as avaliações, assim como, outros podem ter o intervalo de dois anos e meio.

Apesar das limitaçóes, este estudo destaca como pontos relevantes, além do tamanho da amostra utilizada, o fato de terem sido realizadas duas medidas em um grupo de estudantes. Esta ação possibilitou perceber alteraçóes em determinados fatores de risco e a magnitude em que tais alteraçóes ocorreram. Este desenho de estudo é escasso na literatura nacional.

Estes resultados podem auxiliar no entendimento da dinâmica dos FR durante a graduação, bem como na relação com a prática de AF. Assim, estes dados podem embasar açóes institucionais, para além de campanhas de esclarecimento sobre os FR, mas também em políticas de aumento da prática de $\mathrm{AF}$ no ambiente universitário. Visto que, os estudantes passam boa parte de seu tempo diário dentro das instituições.

Diante dos resultados desta pesquisa, foi possível perceber que, menos da metade dos estudantes foram classificados como ativos no início do estudo, não foram alteradas as prevalências dos que cumpriram as recomendaçóes de prática de AF no período de 2011 a 2014. Entre os fatores mais associados com AF, em ambos os períodos, estiveram o consumo recomendado de frutas, saladas e/ou vegetais e a circunferência de cintura elevada. A FAP demonstrou que a contribuição atribuída à prática de $\mathrm{AF}$ foram maiores nas associaçóes com o consumo recomendado de frutas, saladas e/ou vegetais e circunferência de cintura elevada.

\section{Abstract}

Risk factors associated with physical activity practice of college students at the beginning and end of graduation

This descriptive longitudinal study, with 1,197 college students proposed to verify: a) changes in physical activity of college students after four years of course; $b$ ) association of the practice of moderate-to-vigorous physical activity (MVPA) and of vigorous physical activity (AFV) with cardiovascular risk factors (CRF) assessed at the beginning and end of the undergraduation course; $c$ ) fraction attributed to the practice of MVPA and AFV in association with CRF. Through questionnaires International Physical Activity Questionnaire and Youth Risk Behavior Survey, were obtained measures of physical activity practice and of the following FRC: drinking, smoking and eating habits. In addition, were verified: blood pressure, BMI and waist circumference (WC). Through the prevalence ratio was identified the association of MVPA and AFV with CRF. No difference was found in the practice of MVPA, 46.4\% in 2011 and 50.3\% in 2014, as well as for VPA, 30.7\% in 2011 and $35.2 \%$ in 2014 . There was a positive relation of the practice of physical activity with the consumption of alcohol, fruits, salad and/or vegetables, and a protective relation with the high WC, in 2011 and 2014 
$(p<0.05)$. The physical activity was associated with some CRF in both periods. Among the main CRF associated were the recommended intake of fruits, salad and/or vegetables and high measure of waist circumference. Other risk factors may be associated with different environmental variables.

KEYwoRds: Students; Exercise; Risk Factors; Lifestyle.

\section{Referências}

1. World Health Organization. Prevention of cardiovascular disease : guidelines for assessment and management of total cardiovascular risk. Geneva: WHO, 2007.

2. Rech CR, Araújo EDS, Vanat JR. Autopercepção da imagem corporal em estudantes do curso de educaçáo física. Rev Bras Educ Fís Esporte. 2010;24(2):285-92.

3. Farias Júnior JC, Siqueira FV, Nahas MV, Barros MVG. Prevalência e fatores associados a níveis insuficientes de atividade física em jovens estudantes de duas cidades Brasileiras: últimos sete dias e semana típica ou normal. Rev Bras Educ Fís Esporte. 2011;25(4):619-29.

4. Al-Rethaiaa AS, Fahmy AEA, Al-Shwaiyat NM. Obesity and eating habits among college students in Saudi Arabia: a cross sectional study. Nutr J. 2010;9:39.

5. VanKim NA, Laska MN, Ehlinger E, Lust K, Story M. Understanding young adult physical activity, alcohol and tobacco use in community colleges and 4-year post-secondary institutions: A cross-sectional analysis of epidemiological surveillance data. BMC Public Health. 2010;10:208-17.

6. Korhonen T, Kujala UM, Rose RJ, Kaprio J. Physical activity in adolescence as a predictor of alcohol and illicit drug use in early adulthood: a longitudinal population-based twin study. Twin Res Hum Genet. 2009;12(3):261-8.

7. Brandáo, MP, Pimentel FL, Cardoso MF. Impact of academic exposure on health status of university students. Rev Saude Publica. 2011;45(1):49-58.

8. Lloyd-Richardson EE, Bailey S, Fava JL, Wing RA. Prospective Study of Weight Gain During the College Freshman and Sophomore Years. Prev Med. 2009;48(3):256-61.

9. Silva ML, Santos NM, Barnabé V, Valenti VE. Risk factors that may signify a propensity to the use of drugs in students at a public university. Rev Bras Crescimento Desenvolv Hum. 2013;23(3):346-51.

10. World Health Organization. Global Recommendations on Physical Activity for Health. Geneva: WHO; 2010.

11. Feitosa EPS, Dantas CAO, Andrade-Wartha ERS, Marcellini OS, Mendes-Netto RS. Hábitos alimentares de estudantes de uma universidade pública no Nordeste, Brasil. Alim Nutr. 2010;21(2):225-30.

12. Mielke GI, Ramis TR, Habeyche EC, Oliz MM, Tessmer MGS, Azevedo MR, et al. Atividade física e fatores associados em universitários do primeiro ano da universidade federal de Pelotas. Rev Bra Ativ Fís Saúde. 2010;15(1):57-64.

13. Palma A, Abreu RA, Cunha CDA. Comportamentos de risco e vulnerabilidade entre estudantes de Educaçáo Física. Rev Bras Epidemiol. 2007;10:117-26.

14. Anstey KJ, Hofer SM. Longitudinal designs, methods and analysias in Psychiatric Research. Aust N Z J Psychiatry. 2004;38(3):93-104.

15. Gasparotto GS, Gasparotto LPR, Rossi LM, Moreira NB, Bontorin MS, Campos W. Associação entre o período de graduação e fatores de risco cardiovascular em universitários. Rev Lat-Am Enfermagem. 2013;21(3):687-94.

16. Medronho RA. Epidemiologia. 2a ed. São Paulo: Atheneu; 2009.

17. Sousa MH, Silva NN. Estimativas obtidas de um levantamento complexo. Rev Saude Pública. 2003;37(5):662-70.

18. Carvalho AMC, Araújo TME. Fatores associados à cobertura vicinal em adolescentes. Acta Paul Enferm. 2010;23(6):796-802.

19. Martins IS, Velásquez-Meléndes G, Cercato AM. Estado nutricional de grupamentos sociais da área metropolitana de Sáo Paulo. Cad Saúde Pública. 1999;15(1):71-8.

20. Pardini R, Matsudo S, Araújo T, et al. Validação do questionário internacional de nível de atividade física (IPAQ-versão 6): estudo piloto em adultos jovens brasileiros. Rev Bras Ciên Mov. 2001;9(3):45-51.

21. Mello MVO, Bernardelli Junior R, Menossi BRS, Vieira FSF. Risk behavior for the health of students at the State University of North Parana, Brazil-a proposal for online intervention. Cienc Saúde Coletiva. 2014;19(1):159-64.

22. Teixeira M. YRBS-C: Tradução, adaptação transcultural e propriedades psicométricas [dissertação]. Londrina (PR): Escola de Educaçáo Física, Universidade Estadual de Londrina; 2009. 
23. U.S. Department of Agriculture; U.S. Department of Health and Human Services. Dietary Guidelines for Americans. Washington: U.S. Government Printing Office; 2010.

24. Associação Brasileira de Empresas de Pesquisa. Critério padrão de classificação econômica Brasil/2008. São Paulo: ABEP; 2008.

25. Heyward VH, Stolarczyk LM. Applied body composition assessment. Champaign: Human Kinetics; 1996.

26. Wengreen HJ, Moncur C. Change in diet, physical activity, and body weight among young-adults during the transition from high school to college. Nutr J. 2009;8:32-9.

27. Pullman AW, Masters RC, Zalot LC, et al. Effect of the transition from high school to university on anthropometric and lifestyle variables in males. Appl Physiol Nutr Metab. 2009;34(2):162-71.

28. Small M, Bailey-Davis L, Morgan N, Maggs J. Changes in Eating and Physical Activity Behaviors Across Seven Semesters of College: Living On or Off Campus Matters. Health Educ Behav. 2013;40(4):435-41. Epub 2012 Dec 10.

29. Rodrigues ESR, Cheik NC, Mayer AF. Nível de atividade física e tabagismo em universitários. Rev Saúde Pública. 2008;42(4):672-8.

30. Silva ML, Santos NMR, Barnabé V, Valenti VE. Fatores de risco que podem induzir o uso de drogas por estudantes de uma universidade pública. J Hum Growth Dev. 2013;23(3):346-51.

31. Al-Naggar RA, Al-Dubai SA, Al-Naggar TH, Chen R, Al-Jashamy K. Prevalence and Associated Factors of Smoking among Malaysian University Students. Asian Pac J Cancer Prev. 2011;12(3):619-24.

32. Kwan M, Bobko S, Faulkner G, Donnelly P, Cairney J. Sport participation and alcohol and illicit drug use in adolescents and young adults: A systematic review of longitudinal studies. Addict Behav. 2014;39(3):497-506.

33. Peltzer K, Pengpid S, Samuels TA, et al. Prevalence of Overweight/Obesity and Its Associated Factors among University Students from 22 Countries. Int J Environ Res Public Health. 2014;11(7):7425-41.

34. Khalaf A, Ekblom Ö, Kowalski J, Berggren V, Westergren A, Al-Hazzaa H. Female university Students' physical activity levels and associated factors-a cross-sectional study in southwestern Saudi Arabia. Int J Environ Res Public Health. 2013;10(8):3502-17.

35. Santos MC, Angarten VG, Munaro HLR, Pelegrini A. Associação do excesso de peso com variáveis demográficas e atividade física no lazer em universitários. Rev Bras Ciênc Mov. 2014;22(3):14-9.

36. Silva DAS, Quadros TMB, Gordia AP, Petroski EL. Associação do sobrepeso com variáveis sócio-demográficas e estilo de vida em universitários. Cienc Saúde Coletiva. 2011;16(11):4473-9.

37. Hajian-Tilaki K, Heidari B. Is waist circumference a better predictor of diabetes than body mass index or waist-to-height ratio in Iranian adults? Int J Prev Med. 2015;6:5.

38. Carvalho CA, Almeida Fonseca PC, Barbosa JB, Machado SP, Santos AM, Silva AAM. Associaçáo entre fatores de risco cardiovascular e indicadores antropométricos de obesidade em universitários de São Luís, Maranhão, Brasil. Cienc Saúde Coletiva. 2015;20(2):479-90.

\begin{tabular}{r|l} 
ENDEREÇo & Recebido para publicação: 31/05/2016 \\
Guilherme da Silva Gasparotto & $1^{\text {a }}$ revisão: 07/09/2016 \\
Rua João Negrão, 1285 - Rebouças & $2^{\text {a }}$ revisão: 04/11/2016 \\
80230-150 - Curitiba - Paraná - BRASIL & Aceito: 26/01/2017 \\
e-mail: guilherme.gasparotto@ifpr.edu.br &
\end{tabular}

